\title{
Posterior Wall Colon Half Anastomosis for Fecal Diversion; a Pilot Prospective Study
}

\author{
Mohamed KF Hamed, MD; Ahmed M Sabry, MD; Amr H Afifi, MD \\ Department of General Surgery, Faculty of Medicine, Ain Shams University, Egypt
}

Background: Colonic diversion is a common procedure in the practice of colo-rectal surgery; whether on emergent or elective basis. This "diversion", taking place after colon resection when primary anastomosis is "risky", takes one of two forms i.e divided or loop form. We introduced a modification for the double-barrel colostomy technique into a hand-sewn loop colostomy at the two divided ends achieving fecal diversion and at the same time assessing the healing power in the patient during the initial operation.

Patients and method: Fifty patients, candidate for fecal diversion after right colonic resection, having different risk factors for anastomotic leakage, were recruited. A posterior wall half colonic anastomosis was done between both resection ends before exteriorization in those patients instead of performing an ileostomy and a mucus fistula. Patients, then, completed their treatment plan for the primary pathology and their stomas were closed lately as "a loop" ileocolostomy without the need for re-laparotomy.

Results: All patients (with the except of one patient) had uneventful postoperative course following fecal diversion using the "hand-sewn loop" technique as regards bowl viability and fixation with all the patients being lately closed successfully without the need for re-laparotomy.

Conclusion: Performing a posterior wall half-anastomosis at the initial operation of fecal diversion is a feasible option for patients undergoing fecal diversion after partial colonic resection, helping to avoid morbidities encountered during the operation of restoration of bowl continuity while, simultaneously, testing for hidden risks for anastomotic leakage.

Key words: Fecal diversion, hand-sewn loop colostomy, colonic half anastomosis.

\section{Introduction}

Colon diversion or fecal diversion as preferred by many authors, is a common procedure in the practice of colorectal surgeons that may even be a life-saving procedure in some circumstances. ${ }^{1}$

Following colonic resection, fecal diversion is still adopted in the modern surgical era in order to avoid a dreadful complication of primary colonic anastomosis in certain circumstances; anastomotic leak and fecal peritonitis 2,3 with their significant morbidity and mortality. ${ }^{4}$ Anastomotic leakage is assumed to be the leading cause of mortality after colo-rectal surgery. ${ }^{4}$

Many definitions for anastomotic leakage were suggested in the literature. However, the most detailed and practical definition includes a group of clinical manifestations like fever and peritonitis; biochemical indicators as leucocytosis; and various radiologic studies documenting fluid/ gas collections ${ }^{5}$ indicating a connection between the intra- and extraluminal compartments. ${ }^{6}$

Colostomies can be regarded as one of two forms: loop and divided colostomies. ${ }^{7}$ While fixing an opened intestinal loop to the abdominal wall without complete transection in loop colostomy, a divided colostomy requires complete separation of the bowel loop resulting in a proximal colostomy and separated distal mucous fistula (or a blind end pouch in case of Hartman's) sutured to the abdominal wall through separate incisions (or one large aperture in case of double-barrel forms). ${ }^{8}$

The aim of this study is to perform posterior wall anastomosis (i.e. half anastomosis) between both ends creating a "hand sewn loop colostomy" between the remaining two ends of the colon after colonic resection i.e. transferring the double-barrel colostomy into a hand-sewn loop colostomy aiming to facilitate the second operation of bowl continuity restoration and at the same time, testing for the healing power of colonic wall on patient individual basis.

\section{Patients and methods}

Thirty (30) patients undergoing right hemicolectomy and temporary fecal diversion for different indications; whether emergency and elective, at the Ain Shams University Hospitals, were included in this pilot prospective study after taking the approval of "the ethical committee for scientific research of the general surgery department". No pre-requisites 
were needed to incorporate patients in this study provided they were fit for anaesthesia and temporary fecal diversion was indicated. However, we excluded cases with mesenteric vascular insults (mostly they would need a second-look laparotomy and the healing power in those patients is already impaired). We, also, excluded patients with history of previous laparotomy from our study (colon exteriorization may be difficult, requiring extensive dissection due to frozen abdomen). Patients with unresectable colon pathology (discovered intra-operatively), were excluded and replaced with another patients, as well (Those patients are candidates for diversion without colectomy).

The demographic data of all patients were recorded, besides all relevant medical data concerning age, sex, comorbidities and body-mass index (BMI) as well as the indication for diversion.

Having finished the previously decided operation of right hemicolectomy, both ends of the remaining colon were sutured together performing posterior wall anastomosis using vicryl 2/0 and single layer extra-mucosal simple continuous suturing technique (with the mesenteric border at the midline). The posterior wall was defined as about half the circumference of the bowl with the mesenteric border taken as the meridian around which the sutures would go on both sides. Both ends were then exteriorized as a loop colostomy with nippling of the ileal end, from an aperture about $2 \mathrm{~cm}$ in the abdominal wall under no tension (whether at a trans-rectus or a para-rectus position i.e. at about 3-5 cm lateral to the lateral edge of rectus abdominis muscle). Maturation of those "loop ileocolostomies" was done using simple interrupted $2 / 0$ vicryl sutures taking colonic wall full thickness and the dermis of abdominal wall skin. Nippling of the ileal end was done by passing those "maturation stitches" through the seromuscular layer as an "intermediate step". (Figures 1-3).

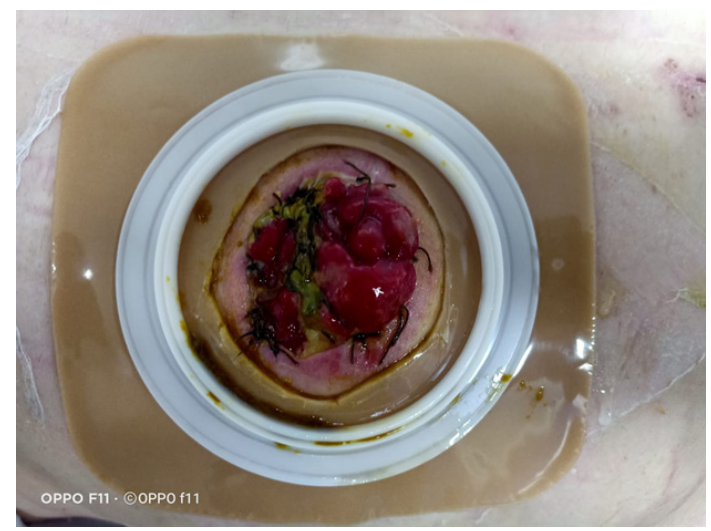

Fig 1

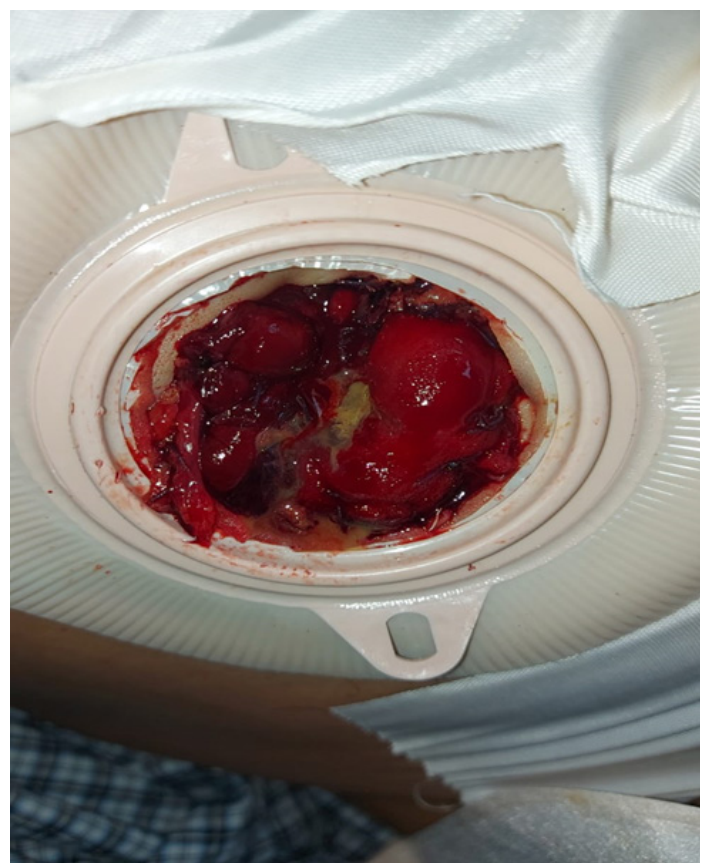

Fig 2

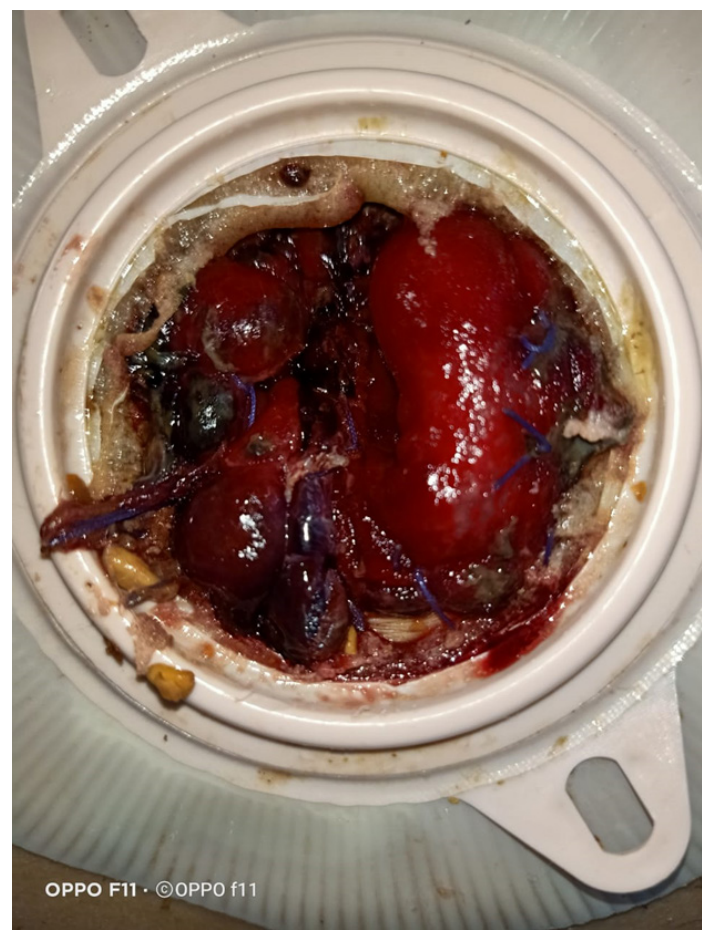

Fig 3

It is important to state that those patients were candidates for temporary fecal diversion due to generalized debilitation (in cases of elective hemicolectomies for cancer) and generalized sepsis (in cases of emergency diversion due to fecal peritonitis).

All patients were allowed to start oral feeding once the stoma was functioning (within the first 48 hours) then discharged. Patients were then followed-up in the out-patient clinic twice-monthly in the first month then once monthly till patient was ready for 
restoration of bowl continuity after finishing the treatment protocol specific for the indication for colectomy.

Patients, ready for bowl continuity restoration, underwent distal loopogram pre-operatively to ensure distal bowl lumen patency and then the colostomy was taken down and dealt with as a loop colostomy i.e. anterior wall of the colonic loop was anastomosed using 2/0 vicryl stitches in a simple continuous extra-mucosal pattern without having to perform a "second-stage laparotomy".

\section{Results}

Thirty patients undergoing temporary fecal diversion after right hemicolectomy were recruited in this study from September 2018 till October 2019 including 17 males and 13 females. The demographic data of the recruited patients are shown in Table $\mathbf{1}$ with the co-morbidities found in those patients enumerated in Table 2.

The indication for colectomy and fecal diversion were variable having 23 patients being operated upon as an elective case while 7 patients were presented to the emergency department. Table 3.

Of the total number of recruited patients, there were thirteen patients who underwent extended right hemicolectomy. The rest of cases (17) underwent formal right hemicolectomy preserving both branches of the middle colic artery. Table 4.
The stoma was exteriorized in a trans-rectus position in 20 patients, while in the rest of cases, the stoma was fashioned in a para-rectus position (10 patients). Table 4.

During the postoperative period, all cases passed uneventful course with the exception of one case that developed muco-cutaneous dehiscence on postoperative day 1 , followed by the development of stomal retraction within the subcutaneous plane leading to localized peri-stomal sepsis and finally para-stomal hernia on day 45 . The data of that case was re-analyzed and is shown in Table $\mathbf{5}$. This case was managed conservatively with frequent dressing of the wound (using saline $0.9 \%$ and povidone iodine daily) and correcting associated laboratory abnormalities (anemia and hypoalbuminemia) until the patient was ready for restoration of bowl continuity and repair of the associated hernia (without application of mesh).

All cases were closed later on after completing their specific treatment plan without the need for relaparotomy.

It is worth to mention that no case was encountered with postoperative intestinal obstruction and the stoma was viable in all cases beginning to function within the first 48 hours during the first operation while the colon regained function around day 3 after the second operation i.e. restoration of bowl continuity.

Table 1: Demographic data of included patients

\begin{tabular}{llll}
\hline Variable & & & Percentage (Total= 50) \\
\hline & Male & & $57 \%$ \\
Sex & Female & 17 & $43 \%$ \\
& & 13 & $63 \%$ \\
Age & $20-39$ yrs & 19 & $37 \%$ \\
& $40-60$ yrs & 11 & $37 \%$ \\
BMI & & $47 \%$ \\
& $<30$ & 11 & $17 \%$ \\
& $30-35$ & 14 & $63 \%$ \\
Occupation & $>35$ & 5 & $37 \%$ \\
\hline
\end{tabular}

Table 2: Co-morbidities of patients

\begin{tabular}{ll}
\hline Co-morbidities (19 out of $\mathbf{3 0}$ patients) & \\
\hline Diabetic & 12 \\
Asthmatic & 3 \\
Smoker & 9 \\
\hline
\end{tabular}


Table 3: Indication of diversion

\begin{tabular}{lll}
\hline Emergency cases & Total $=7$ (23\%) \\
& Stab abdomen & 1 (extended RT colectomy) \\
Perforated cancer & 4 (3 extended and 1 formal RT) \\
Iatrogenic injury & 2 (Formal RT) \\
Elective cases & Total $=23(77 \%)$ \\
& Rt colectomy & 14 \\
& Extended RT colectomy & 9 \\
\hline
\end{tabular}

Table 4: Site of stomas in relation to rectus abdominis muscle

\begin{tabular}{|c|c|c|c|c|}
\hline \multirow[t]{2}{*}{ Operation done } & \multirow[b]{2}{*}{ Right hemicolectomy } & \multicolumn{2}{|c|}{ Number $(n=50)$} & \multirow{2}{*}{$\begin{array}{l}\text { Percentage } \\
57 \%\end{array}$} \\
\hline & & 17 & & \\
\hline & & $\begin{array}{l}\text { Trans-rectus } \\
\text { stoma= } 11\end{array}$ & $\begin{array}{l}\text { Para-rectus } \\
\text { stoma }=6\end{array}$ & \\
\hline & Extended right hemicolectomy & 13 & & $43 \%$ \\
\hline & & $\begin{array}{l}\text { Trans-rectus } \\
\text { stoma }=9\end{array}$ & $\begin{array}{l}\text { Para-rectus } \\
\text { stoma }=4\end{array}$ & \\
\hline \multirow[t]{2}{*}{ Site of stoma } & Trans-rectus & 20 & & $67 \%$ \\
\hline & Para-rectus & 10 & & $33 \%$ \\
\hline
\end{tabular}

Table 5: Data of complicated patient

\begin{tabular}{ll}
\hline Age & 56 years \\
Co-morbidities & COPD (smoker), diabetic (uncontrolled) \\
BMI & 37 \\
Operation & Left Hemicolectomy \\
Final diagnosis & Perforated splenic flexure cancer \\
Stoma & Trans-rectus \\
Pre-operative Hb & $9 \mathrm{gm} / \mathrm{l}$ \\
Pre-operative Albumin & $2.3 \mathrm{gm} / \mathrm{l}$ \\
\hline
\end{tabular}

\section{Discussion}

Fecal diversion following hemicolectomy is a common procedure in the career of surgeons and a life-saving procedure in many situations. However, this procedure has a strong impact on patient's wellbeing, physically and psychologically, ${ }^{9}$ and the restoration of bowel continuity "taking down that stoma" is full of complications; either due to the patient's co-morbidities or iatrogenic injuries during the re-laparotomy in a "non-virgin" abdomen. ${ }^{10}$

The diverting stoma can take one of two forms: either a loop form or an end form with the loop form having the advantage of easier and shorter reversal time during the restoration of bowel continuity and fewer reversal related complications. ${ }^{11}$
The idea of the study was decided after performing a retrospective cohort study of the medical records of our patients in Ain Shams University hospital reaching the result that "hand-sewn" loop colostomies have many advantages in comparison to the classic separate colostomies (or ileostomies) and mucus fistulae after hemicolectomies. ${ }^{12}$

Thirty patients, with various risk factors for primary anastomotic leakage and consequently stoma complications, were included in this study. For the sake of facilitating data interpretation, the patients were viewed as two subsets i.e. those below the age of 40 years and those above 40 years. Also, they were viewed as patients having a para-rectus stoma and those having a trans-rectus one. Obesity, as a risk factor for stoma complications, was also 
studied; seeing patients as those with body mass index (BMI) less than 30, those with BMI 30:35 and those with BMI above $35 .{ }^{13}$

Only patients with right colonic lesions were included for the proposed procedure to be done with one of the ends is the ileum aiming to decrease the size of the abdominal wall aperture needed for stoma exteriorization (for fear of parastomal hernia development while studying the proposed technique which may affect the stoma viability and functionality).

The site of the stoma exteriorization was left for the surgeon's preference, whether at the lateral edge of the rectus muscle (trans-rectus) or away from it (para-rectus). Many studies proved the site of the stoma maturation has no impact on the development of para-stomal hernia. ${ }^{13-15}$ This point i.e. the site of stoma exteriorization, is still a matter of debate. Many authors assumed that a para-rectus stoma is a risk factor for para-stomal hernia. ${ }^{16}$ In our study, no difference was noted as regards the incidence of para-stomal hernia development in accordance with the study of Hardt et al. ${ }^{15}$

One of the main issues of concern regarding the loop stomas is the need for having a relatively large aperture in the abdominal wall for exteriorization of such stomas, raising the risk of para-stomal hernia occurrence or even stomal prolapse., ${ }^{7,17}$

In our study, we had only one case of para-stomal hernia that developed on post-operative day 45 . However, on reanalyzing the data of that patient, such complication could be attributed to the postoperative sepsis developed in that patient due to several co-morbidities (morbid obesity, uncontrolled diabetes, anaemia and hypo-albuminemia) that couldn' $t$ be corrected pre-operatively because of the emergency indication of that operation (acute abdomen due to perforated cecal cancer). The rest of the thirty patients had an uneventful postoperative course i.e. without the development of stomal prolapse. This doesn ' $t$ go with the findings of Seamon et al, ${ }^{17}$ Youssef et $\mathrm{al}^{7}$ and Krishnamurty et al. ${ }^{18}$

It is worth to mention that the previous stated studies were either a retrospective study ${ }^{7,17}$ or a case report. ${ }^{18}$ On the other hand, our study has two main limitations being a pilot study i.e. the relatively small number of patients (only fifty candidates) and the short period of follow-up (three months on average as those stomas were temporary ones). This point is worth to be a subject of future studies to determine the actual relationship between loop colostomies on one hand with the para-stomal hernias and stomal prolapse on the other hand.

Stomal ischemia is an important point; having to pass two loops of the colon (small intestine and colon in cases after right hemicolectomy) through a single opening which is essentially associated with mechanical trauma and compression of the small mesenteric venules at the level of abdominal wall aperture..$^{19}$ On the other hand, increasing the diameter of the aperture beyond three centimeters (or the size allowing the passage of two fingers of the surgeon) is a risk factor for the development of para-stomal hernia. ${ }^{20}$

In our study, we didn ' $t$ encounter that problem with stoma viability; sticking to sound surgical techniques when the operations were all done by the same surgical team. This goes in accordance with the results reached by Manley et al ${ }^{11}$ comparing loop to end colostomies for fecal diversion in cases of colonic injuries.

In our institute, it is uncommon to use a rod support for loop colostomies as it is believed to have no significant role in preventing stoma retraction; having exteriorized the stoma under no tension and in the same time maintaining adequate vascularity for the exteriorized segment attaching to sound surgical techniques and performing adequate dissection within the surgical planed as needed. This is exactly the same recommendations of the Multidisciplinary Italian Study group for STOmas (MISSTO). ${ }^{13}$

The proposed technique of performing posterior wall "half-anatomosis" can be viewed as a pretest for the healing power in "colectomy" patients before actually testing that on the patient having restored the bowl continuity, taking down the stoma performed. Having both ends of the colon (or ileum in cases of right hemi-colectomy) exteriorized, allows for full diversion if leakage occurred "extraperitoneally" in that sample anastomosis.

Being a pilot study, despite being a prospective one, poses limitations on this study. The idea of performing a hand-sewn loop colostomy with two separate bowl segments (or even using staplers in a side to side fashion) is appealing especially in patients with co-morbidities, eliminating the need for re-laparotomy for stoma take-down and restoration of bowel continuity. However, this cannot be recommended by studying a relatively small number of patients (30 patients). Further study of that option on a larger number of patients is recommended.

In our study, we only included patients with right colonic lesions to have the aperture of the stoma in the abdominal wall as small as possible for fear of developing parastomal hernia. Whether this technique can be applied to those with other colonic lesions i.e., transverse, left, sigmoid colon and rectum needs to be studied separately. 
The effect of chemotherapy and/or radiotherapy on the viability of such stomas is another issue to be studied in future research on a wider range, taking in consideration that chemo and radiotherapy in general, affect cellular proliferation and vascularity of colonic mucosa; both of which are essential for the "half anastomosis" done in the posterior wall. However, this aspect can be a point of strength in favor of our proposed technique, at least theoretically. Performing a posterior wall "halfanastomosis" allows for assessment of the healing power of the tissues generally and colonic wall specifically in such patients before performing the whole anastomosis intraperitoneally; thus, avoiding reducing the risk of anastomotic leakage in such frail patients.

\section{Conclusion}

Performing a "hand-sewn" loop colostomy (or ileocolostomy) during the first operation of hemicolectomy is a feasible option facilitating the restoration of bowel continuity in cases of temporary diversion by eliminating the need for a relaparotomy

Disclosure and conflict of interest: The authors have no disclosure and no conflict of interest.

\section{References}

1. Shazi $B$, Bruce JL, Laing $G L$, et al: The management of colonic trauma in the damage control era. Ann R Coll Surg Engl. 2017; 99(1): 76-81.

2. Slieker JC, Daams F, Mulder IM, et al: Systematic review of the technique of colorectal anastomosis. JAMA Surg. 2013; 148(2): 190201.

3. Paral J, Lochman P, Blazej $S$, et al: Glued versus stapled anastomosis of the colon: An experimental study to determine comparative resistance to intraluminal. Asian Journal of Surgery. 2014; 37: 154-161.

4. Daams F, Luyer $M$, Lange JF: Colorectal anastomotic leakage: Aspects of prevention, detection and treatment. World J Gastroenterol. 2013; 19(15): 2293-2297.

5. Kingham TP, Pachter HL: Colonic anastomotic Leak: Risk factors, diagnosis and treatment. J Am Coll Surg. 2009; 208(2): 269-278.

6. Park JS, Huh JW, Park YA, et al: Risk factors of anastomotic leakage and long-term survival after colorectal surgery. Medicine (Baltimore). 2016; 95(8): e2890.

7. Youssef F, Arbash G, Puligandla PS, et al: Loop versus divided colostomy for the management of anorectal malformations: A systematic review and meta-analysis. J ped Surg. 2017; 52(5): 783-90.

8. Askarpour S, Peyvasteh M, Changai B, et al: Skin bridge versus rod colostomy in childrencomparison between complications. Pol Przegl Chir. 2012; 84(10): 485-487.

9. De Campos K, Bot LHB, Petroianu A, et al: The impact of colostomy on the patient's life. Journal of Coloproct. 2017; 37(3): 205-210.

10. Fonseca AZ, Uramoto $E$, Santos-Rosa OM, et al: Colostomy closure: Risk factors for complications. Arq Bras Cir Dig. 2017; 30(4): 231-234.

11. Manley NR, Sharpe JP, Lewis RH, et al: Analysis of over 2 decades of colon injuries identifies optimal method of diversion: Does an end justify the means? J Trauma Acute Care Surg. 2019; 86(2): 214-219.

12. Farghaly M, Youssef T: Post-right hemicolectomy ileostomy and mucous fistula through single skin opening: Comparative study with ileostomy and mucous fistula through two skin openings. Egyptian Journal of Surgery. 2019; 38(4): 691699.

13. Ferrara $F$, Parini $D$, Bondurri $A$, et al: Multidisciplinary Italian study group for Stomas (MISSTO) Italian guidelines for the surgical management of enteral stomas in adults. Tech Coloproctol. 2019; 23(11): 1037-1056.

14. Li W, Benlice C, Stocchi L, et al: Does stoma site specimen extraction increase postoperative ileostomy complication rates? Surg Endosc. 2017; 31: 3552-3558.

15. Hardt J, Seyfried S, Weiß C, et al: A pilot singlecentre randomized trial assessing the safety and efficacy of lateral pararectus abdominis compared with transrectus abdominis muscle stoma placement in patients with temporary loop ileostomies; the PATRASTOM trial. Colorectal Dis. 2016; 18(2): 81-90.

16. Novell R, Li MKW, Ogunbiyi O, et al: Transverse and sigmoid colostomies in colon. In Kirk's general surgical operations, sixth edition. 2013: 230:232, Elsevier.

17. Seamon LG, Richardson $D L$, Pierce $M$, et al: Local correction of extreme stomal prolapse following transverse loop colostomy. Gynecol Oncol. 2008; 111(3): 549-51.

18. Krishnamurty DM, Blatnik J, Mutch M: Stoma complications. Clin Colon Rectal Surg. 2017; 
30(3): 193-200.

19. Beck DE: Ostomy Construction and Management: Personalizing the Stoma for the Patient. In Shackelford's Surgery of the Alimentary Tract. Eighth Edition, Elsevier. 2019;
Chapter 178: 2147-2162.

20. Styliński R, Alzubedi A, Rudzki S: Parastomal hernia; current knowledge and treatment. Videosurgery Miniinv. 2018; 13(1): 1-8. 